The phase transition and elastic and optical properties of polymorphs of Cul

This article has been downloaded from IOPscience. Please scroll down to see the full text article.

2012 J. Phys.: Condens. Matter 24475503

(http://iopscience.iop.org/0953-8984/24/47/475503)

View the table of contents for this issue, or go to the journal homepage for more

Download details:

IP Address: 141.219.155.120

The article was downloaded on 30/10/2012 at 16:08

Please note that terms and conditions apply. 


\title{
The phase transition and elastic and optical properties of polymorphs of $\mathrm{CuI}$
}

\author{
Jiajie Zhu ${ }^{1,3}$, Ravindra Pandey ${ }^{1}$ and $\mathrm{Mu} \mathbf{G u}^{2}$ \\ ${ }^{1}$ Department of Physics, Michigan Technological University, Houghton, MI 49931, USA \\ ${ }^{2}$ Department of Physics, Tongji University, Shanghai 200092, People's Republic of China \\ E-mail: pandey@mtu.edu
}

Received 4 May 2012, in final form 10 October 2012

Published 26 October 2012

Online at stacks.iop.org/JPhysCM/24/475503

\begin{abstract}
The high-pressure polymorphs of $\mathrm{CuI}$ have attracted much attention due to the somewhat contradictory identification of their structures by means of x-ray diffraction measurements and theoretical calculations. In this paper, we report the results of a theoretical investigation of polymorphs of $\mathrm{CuI}$ including zinc-blende, rhombohedral, tetragonal, rocksalt and orthorhombic phases. We find that $\mathrm{CuI}$ follows the high-pressure transition path from the zinc-blende phase to the orthorhombic phase via the tetragonal phase, and the rhombohedral phase shows mechanical instability under high pressure. The bulk moduli are almost isotropic but the shear moduli show large anisotropy in these polymorphs. A relatively strong hybridization of $\mathrm{I} \mathrm{p}$ and $\mathrm{Cu}$ d states appears to determine the electronic properties of the $\mathrm{CuI}$ polymorphs. The zinc-blende and tetragonal $\mathrm{CuI}$ are direct gap semiconductors and their optical properties are similar, whereas the orthorhombic $\mathrm{CuI}$ is metallic.
\end{abstract}

S Online supplementary data available from stacks.iop.org/JPhysCM/24/475503/mmedia

(Some figures may appear in colour only in the online journal)

\section{Introduction}

Cuprous iodine $(\mathrm{CuI})$ is a well-known optical material with many interesting physical and chemical properties such as high ionic conductivity $\left(\approx 0.1 \mathrm{~S} \mathrm{~cm}^{-1}\right)$, wide band gap $(\approx 3.1 \mathrm{eV})$, and large excitonic binding energy $(\approx 62 \mathrm{meV})$ [1-4]. It has also attracted much attention as an ultrafast scintillator due to its fast decay time of about 130 ps [5, 6]. At ambient conditions, CuI occurs in the zinc-blende $(\gamma)$ phase, though it can be transformed into several polymorphs at high temperature and high pressure. At ambient pressure, a high-temperature phase transition from the zinc-blende $(\gamma)$ phase to the hexagonal $(\beta)$ phase between 642 and $680 \mathrm{~K}$, and the cubic $(\alpha)$ phase above $680 \mathrm{~K}$ is reported [7]. The successive high-pressure polymorphs of $\mathrm{CuI}$, on the other hand, are reported to be rhombohedral $(<1.4 \mathrm{GPa})$, tetragonal $(1.4-4.7 \mathrm{GPa})$ and rocksalt $(>10 \mathrm{GPa})$ phases by the experimental studies [8-10]. Surprisingly, theoretical calculations based on

\footnotetext{
3 Permanent Address: Department of Physics, Tongji University, Shanghai, People's Republic of China.
}

density functional theory do not find the high-pressure phase transition from zinc-blende to rhombohedral [11-14]. This is in spite of the fact that first-principles calculations with proven predictive capabilities are now routinely performed on a wide variety of materials to gain an understanding of their structural, thermodynamic, electronic and optical properties [15]. Furthermore, experimental and theoretical investigations have so far only focused on determining a limited set of structural and electronic properties of polymorphs of CuI. These properties include lattice constants, bulk modulus and electronic band structures of polymorphs of CuI. And to the best of our knowledge, a detailed study of elastic and optical properties of these polymorphs including the orthorhombic phase of $\mathrm{CuI}$ has not been performed previously.

In this paper, we will perform a comprehensive theoretical study of polymorphs of CuI. Specifically, we will focus on calculations of elastic constants to examine the stability of the high-pressure rhombohedral phase. Our results will suggest that the rhombohedral phase is not stable, and the phase transition from tetragonal to 
orthorhombic via rocksalt is not preferred. We will also calculate the electronic properties including effective electron mass, optical reflectivity, index of refraction and optical absorption coefficients of the high-pressure polymorphs of CuI.

In section 2, we describe the computational method. In section 3 , we present the results and discussion and give a brief summary in section 4 .

\section{Computational method}

The electronic structure calculations are carried out using the projector augmented plane-wave (PAW) method as implemented in Vienna $a b$ initio simulation package (VASP) [16, 17]. The generalized gradient approximation (GGA) of Perdew, Burke and Ernzerhof (PBE) is adopted for the exchange-correlation potential [18] to the density functional theory (DFT). The $3 \mathrm{~d}$ and $4 \mathrm{~s}$ electrons of $\mathrm{Cu}$ together with $5 \mathrm{~s}$ and $5 \mathrm{p}$ electrons of I are considered as the valance electrons in the calculations.

The Brillouin-zone integrations are performed with the $10 \times 10 \times 10 k$-point mesh for the structural optimization calculations. Later, a $16 \times 16 \times 16 k$-point mesh is used for density of state (DOS) calculations. The cut-off energy for plane-wave basis is $355 \mathrm{eV}$. The energy tolerance is $10^{-6} \mathrm{eV}$ in the iterative solution of the Kohn-Sham equations. The structure and atoms are relaxed until the force on each atom is less than $0.01 \mathrm{eV} \AA^{-1}$. For a larger plane-wave cut-off value of $500 \mathrm{eV}$ with a denser $k$-point mesh of $20 \times 20 \times 20$, the change in total energy is about $0.02 \mathrm{eV} /$ atom. A similar variation is predicted for the exchange-correlation functional form at the GGA-DFT level of theory. Thus, the residual numerical uncertainty is estimated to be about $0.04 \mathrm{eV} /$ atom in our calculations.

The elastic constants are determined using second-order derivatives of equilibrium energy with respect to strains via

$$
C_{\alpha \beta}=\frac{1}{V} \frac{\partial^{2} E}{\partial \gamma_{\alpha} \partial \gamma_{\beta}},
$$

where $\gamma$ s are applied strains in Voigt's notion around the equilibrium position [19]. The deformations of polymorphs of $\mathrm{CuI}$ depend on their space groups.

The imaginary part of dielectric function, $\varepsilon_{2}(\omega)$, is determined by the summation over occupied states in the valence band and unoccupied states in the conduction band using the equation:

$$
\begin{gathered}
\varepsilon_{2}^{\alpha \beta}(\omega)=\frac{4 \pi^{2} e^{2}}{V} \lim _{q \rightarrow 0} \frac{1}{q^{2}} \sum_{c, v, k} 2 \omega_{k} \delta\left(\epsilon_{c k}-\epsilon_{v k}-\omega\right) \\
\times\left\langle u_{c k+e_{\alpha} q} \mid u_{v k}\right\rangle\left\langle u_{c k+e_{\beta} q} \mid u_{v k}\right\rangle^{*}
\end{gathered}
$$

where the index $v$ and $c$ refer to the valence band and conduction band and $u_{c k}$ is the cell periodic part of wave function at different $k$-point [20]. The real part of dielectric function is obtained from Kramers-Kronig transformation via

$$
\varepsilon_{1}(\omega)=1+\frac{2}{\pi} \int_{0}^{\infty} \frac{\omega^{\prime} \varepsilon_{2}\left(\omega^{\prime}\right) \mathrm{d} \omega^{\prime}}{\left(\omega^{\prime 2}-\omega^{2}\right)} .
$$
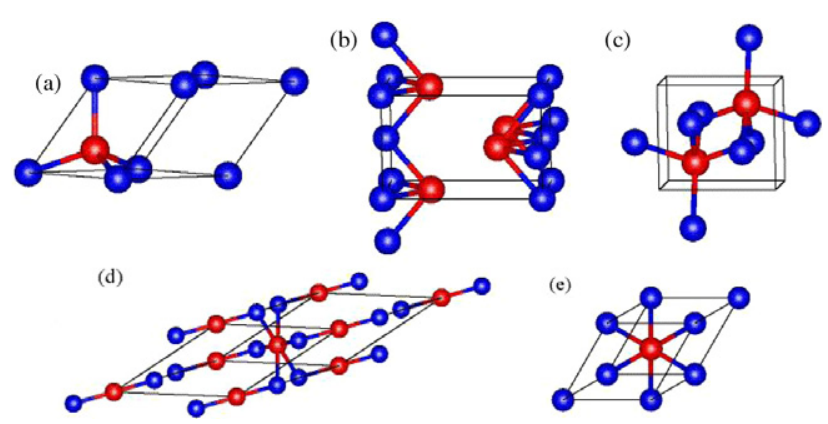

Figure 1. A schematic diagram of polymorphs of CuI: (a) zinc-blende, (b) tetragonal, (c) orthorhombic, (d) rhombohedral and (e) rocksalt.

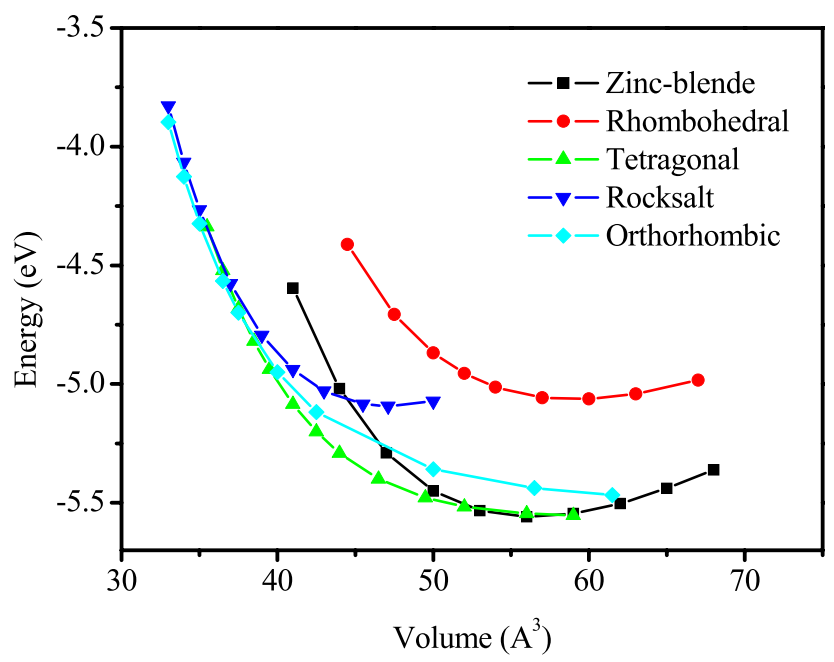

Figure 2. Total energy/formula unit as a function of volume for polymorphs of $\mathrm{CuI}$.

The refractive index is calculated by

$$
n(\omega)=\left(\frac{\sqrt{\varepsilon_{1}^{2}(\omega)+\varepsilon_{2}^{2}(\omega)}+\varepsilon_{1}(\omega)}{2}\right)^{2} .
$$

\section{Results and discussion}

\subsection{Structural properties}

The crystal structures of the polymorphs of $\mathrm{CuI}$ are illustrated in figure 1 . The calculated energy surface of these structures is given in figure 2 where the total energy of per $\mathrm{CuI}$ formula unit is plotted as a function of the crystallographic unit-cell volume of the several polymorphs of CuI. The lattice parameters as well as the internal coordinates are optimized at each fixed value of unit-cell volume. The zinc-blende phase is predicted to be the most stable phase as also experimentally observed. It is followed by the tetragonal phase with a noticeable contracted equilibrium volume $(\approx 10 \%)$ and the energy difference of about $0.06 \mathrm{eV}$.

It is generally understood that a common-tangent construction for the energy versus volume curves of two 
Table 1. The calculated structural properties of polymorphs of CuI at the GGA-DFT level of theory.

\begin{tabular}{|c|c|c|c|c|c|c|}
\hline & \multirow[b]{2}{*}{ Space group } & \multicolumn{2}{|c|}{ Lattice constant $(\AA)$} & \multirow{2}{*}{$\frac{\text { Volume/unit cell }\left(\AA^{3}\right)}{\text { This work }}$} & \multirow{2}{*}{$\begin{array}{l}\text { Cohesive energy (eV) } \\
\text { This work }\end{array}$} & \multirow{2}{*}{$\begin{array}{l}\text { Bond distance } \\
R(\mathrm{Cu}-\mathrm{I})(\AA) \\
\text { This work }\end{array}$} \\
\hline & & This work & Expt $[8,22]$ & & & \\
\hline Zinc-blende & 216 & $a=6.074$ & $a=6.054$ & 56.0 & 5.56 & 2.63 \\
\hline Rhombohedral & 166 & $\begin{array}{l}a=4.452 \\
c=20.49\end{array}$ & $\begin{array}{l}a=4.155 \\
c=20.48\end{array}$ & 58.6 & 5.06 & 2.72 \\
\hline Tetragonal & 129 & $\begin{array}{l}a=4.063 \\
c=6.206\end{array}$ & $\begin{array}{l}a=4.023 \\
c=5.651\end{array}$ & 50.6 & 5.5 & 2.65 \\
\hline Rocksalt & 225 & $a=5.733$ & & 47.1 & 5.09 & 2.87 \\
\hline Orthorhombic & 63 & $\begin{array}{l}a=5.419 \\
b=6.168 \\
c=4.495\end{array}$ & $\begin{array}{l}a=5.373 \\
b=6.115 \\
c=4.609\end{array}$ & 37.5 & 4.69 & 2.53 \\
\hline
\end{tabular}

phases of a system yields the high-pressure transitional pressure. Following this guideline, figure 2 suggests that there exists no phase transition between the zinc-blende and the rhombohedral phase of CuI. The energy difference between these two phases is calculated to be about $0.5 \mathrm{eV}$. Likewise, calculations suggest that there appears to be a phase transition from the zinc-blende phase to the rocksalt phase, though a possibility of the phase transition from the rocksalt phase to either the tetragonal phase or the orthorhombic phase does not exist in CuI.

The structural parameters including lattice constants together with equilibrium volumes and cohesive energies of $\mathrm{CuI}$ in its ambient and high pressure polymorphs are presented in table 1 . We find that the calculated structural properties of the well-characterized zinc-blende phase are in excellent agreement with the previously reported experimental and theoretical values suggesting the accuracy and reliability of our modeling parameters. For example, the equilibrium volume of the zinc-blende phase is calculated to be $56 \AA^{3}$. The corresponding experimental value is $55.5 \AA^{3}$. For the high-pressure orthorhombic phase, the lattice constant values are 5.419, 6.168 and $4.495 \AA$, as compared to the corresponding experimental values of 5.373, 6.115 and $4.609 \AA$, respectively. The calculated internal coordinates of tetragonal and orthorhombic phases are $(\mathrm{Cu}:(0,0,0), \mathrm{I}:(0$, $0.5,0.276))$, and $(\mathrm{Cu} ;(0,0,0)$, I: $(0,0.5,0.276))$, respectively. The structural parameters associated with the tetragonal and orthorhombic phases are calculated at $2.4 \mathrm{GPa}$ and 19.6 GPa, respectively at which the phase transition from the zinc-blende phase is expected to occur.

The cohesive energy values given in table 1 suggest the predicted order of stability to be zinc-blende $>$ tetragonal $>$ rocksalt $>$ rhombohedral $>$ orthorhombic for $\mathrm{CuI}$. The cohesive energy is defined as difference between the total energy of bulk $\mathrm{CuI}$ and the sum of total energies of $\mathrm{Cu}$ atom and I atom.

A change in the coordination number from four (zinc-blende) to five (orthorhombic) occurs with the pressure and is accompanied by the volume contraction of $33 \%$. The near-neighbor distance $R_{\mathrm{Cu}-\mathrm{I}}$ decreases from $2.63 \AA$ for the zinc-blende phase to $2.53 \AA$ for the orthorhombic phase. As expected, the nature of chemical bonding also changes in going from zinc-blende to tetragonal to orthorhombic as evidenced by the projected electron localization functions (ELFs) in figure 3 [21]. The maximum value of ELF around $\mathrm{Cu}$ ion is about 0.77 in the orthorhombic CuI. Note that the values of ELF vary from 0 to 1, which represents complete delocalization and complete localization respectively.

\subsection{Phase transition}

The transition pressure is defined as the pressure where the enthalpy $H=E+P V$ of two phases are equal. The first phase transition, from zinc-blende to tetragonal, occurs at about $2.4 \mathrm{GPa}$. The volume contraction is about $10 \%$, as indicated in figure 4 . The second phase transition, from tetragonal to orthorhombic is observed at about 19.6 GPa and the corresponding volume contraction is $26 \%$. The phase transition from the tetragonal phase to the orthorhombic phase does not occur via the rocksalt phase. Also, no phase transition from the orthorhombic phase to the rocksalt phase is seen up to $40 \mathrm{GPa}$.

Our theoretical results are in a very good agreement with the experimental results [22], though they do not agree with the results obtained by the atomistic calculations [23]. We believe that the use of the atomistic method which lacks the important exchange and correlation contributions may result in predicting the stability of the rocksalt phase between 10 and $18 \mathrm{GPa}$. Furthermore, our calculations find the total energy of the rocksalt and the orthorhombic phases to be almost degenerate at high pressures (figure 2) suggesting the phase transition to occur at high pressure and high temperature conditions.

A direct phase transition from the zinc-blende to the rocksalt phase at about $9.6 \mathrm{GPa}$ is also predicted. This result is in agreement with the previously theoretical results [12-14]. Note that the phase transition from the zinc-blende phase to the rocksalt phase has been seen in $\mathrm{CuCl}$ and $\mathrm{CuBr}$, though it occurs via intermediate phases less than $10 \mathrm{GPa}$ [8].

\subsection{Elastic properties}

Table 2 lists the calculated elastic properties, including elastic constants, bulk and shear moduli and Debye temperature of polymorphs of CuI. For the well-characterized zinc-blende phase, the calculated elastic properties and Debye temperature 

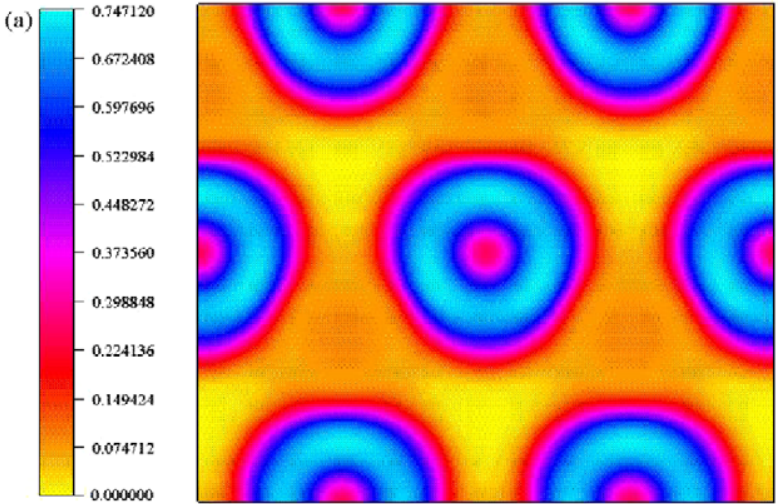

(b)
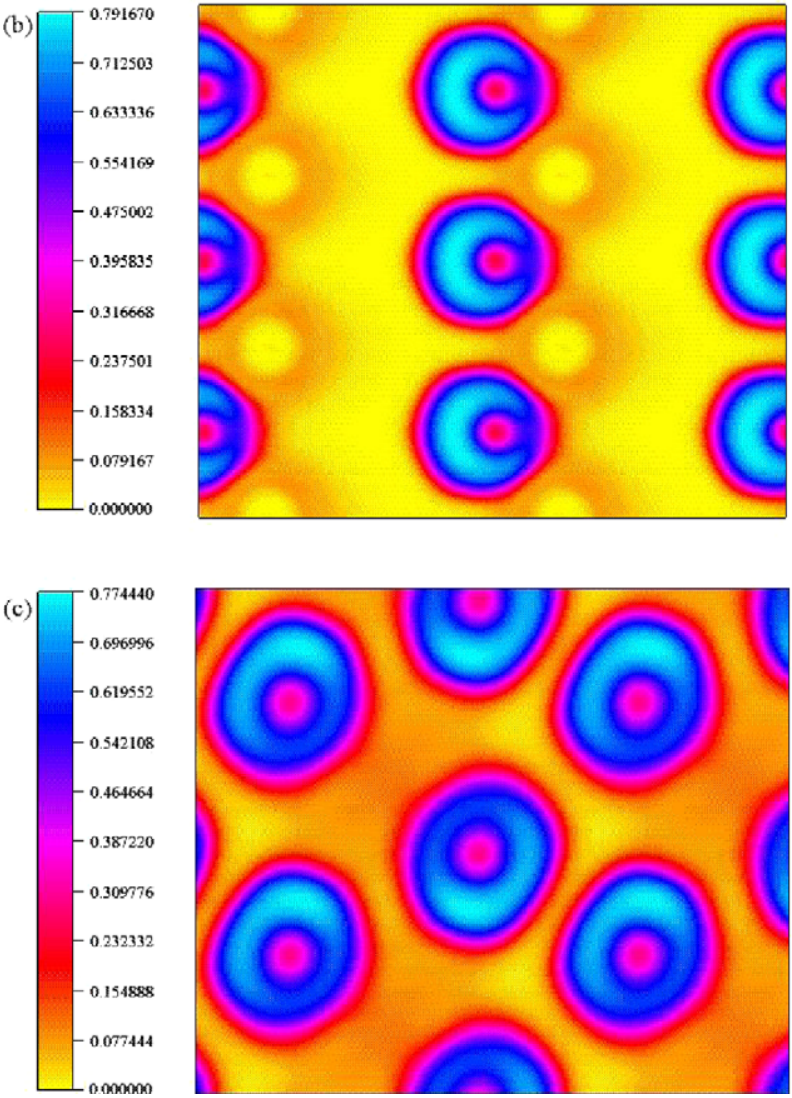

Figure 3. The projected electron localization functions of $\mathrm{CuI}$ : (a) Zinc-blende, (b) tetragonal and (c) orthorhombic.

are in excellent agreement with the corresponding experiment values [24, 25].

The elastic constants of orthorhombic structure are characterized by nine independent components, i.e. $C_{11}, C_{12}$, $C_{13}, C_{22}, C_{23}, C_{33}, \mathrm{C}_{44}, C_{55}$ and $C_{66}$. For tetragonal structure, the independent components reduce to six and they further decrease to three for the zinc-blende structure. $K$ and $G$ are average bulk and shear modulus, respectively [26]. Following the criterion defining the mechanical stability, one expects the elastic constant, $C_{44}$ to be positive for a given material [27]. Although the calculated $C_{44}$ of the tetragonal $\mathrm{CuI}$ satisfies this criterion, it is close to zero suggesting that the tetragonal phase may not be very stable.

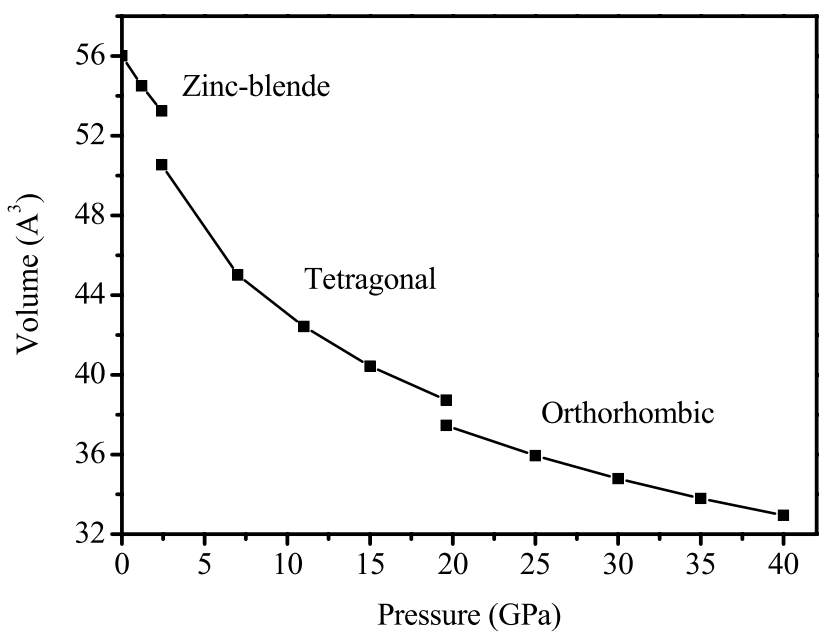

Figure 4. A schematic diagram of the pressure-volume curve for $\mathrm{CuI}$.

We use the subscripts $V, R$ and $H$ to donate Voigt, Reuss and Hill notation, where $K_{H}\left(G_{H}\right)$ is the average value of $K_{V}$ $\left(G_{V}\right)$ and $K_{R}\left(G_{R}\right) . B_{X}$ is the bulk modulus along $x$-axis of a system under hydrostatic pressure. Since dispersion in the directional bulk moduli along different crystal axes reveals the anisotropy of the system, we define the percentage of anisotropy to be $\left(B_{X}-B_{Z}\right) /\left(B_{X}+B_{Z}\right) \times 100 \%$ for $x$ and $z$ direction in the lattice. It is to be noted here that the predicted anisotropy is $18 \%$ for $x$ and $z$ direction in the tetragonal phase but $32 \%$ in the orthorhombic phase. This is due to the fact that $\mathrm{CuI}$ makes a transition from four-coordinated lattice structure to the five-coordinated lattice structure, leading to a strong dependence of lattice constants with pressure in the orthorhombic CuI (figure S1 available at stacks.iop.org/ JPhysCM/24/475503/mmedia).

\subsection{Electronic properties}

The calculated density of states (DOS) and band structures are shown in figures 5 and 6, respectively. For the zinc-blende phase, the uppermost valence band is formed by $\mathrm{Cu} 3 \mathrm{~d}$ and I $5 \mathrm{p}$ states, and the valence band maximum (VBM) is mainly composed of I $5 p$ states with three-fold degeneracy. There is a relatively narrower I 5s band located at $13 \mathrm{eV}$ below VBM. For the tetragonal $\mathrm{CuI}$, I 5 s band now splits into two sub-bands reducing its separation with the upper valence band. For zinc-blende, tetragonal and orthorhombic $\mathrm{CuI}$, the conduction band minimum $(\mathrm{CBM})$ is all composed of the $\mathrm{Cu} 4 \mathrm{~s}$ states with a parabolic character.

The calculations find the zinc-blende to be a direct gap semiconductor with a gap of $1.13 \mathrm{eV}$. It is similar to the values obtained previously [12], though the experimental value is $3.1 \mathrm{eV}$. For the tetragonal phase, the calculated direct gap at $\Gamma$ is $1.54 \mathrm{eV}$ and the indirect $(\Gamma-\mathrm{M})$ gap is $2.35 \mathrm{eV}$. The orthorhombic $\mathrm{CuI}$ shows metallic character due to the crossing of $\mathrm{Cu} 4 \mathrm{~s}$ and I 5p states at the Fermi energy. Here, the $\mathrm{Cu} 3 \mathrm{~d}$ band is pushed down to lower energy away from VBM. It should be pointed out that the GGA-DFT method underestimates the band gap of the semiconducting and ionic 

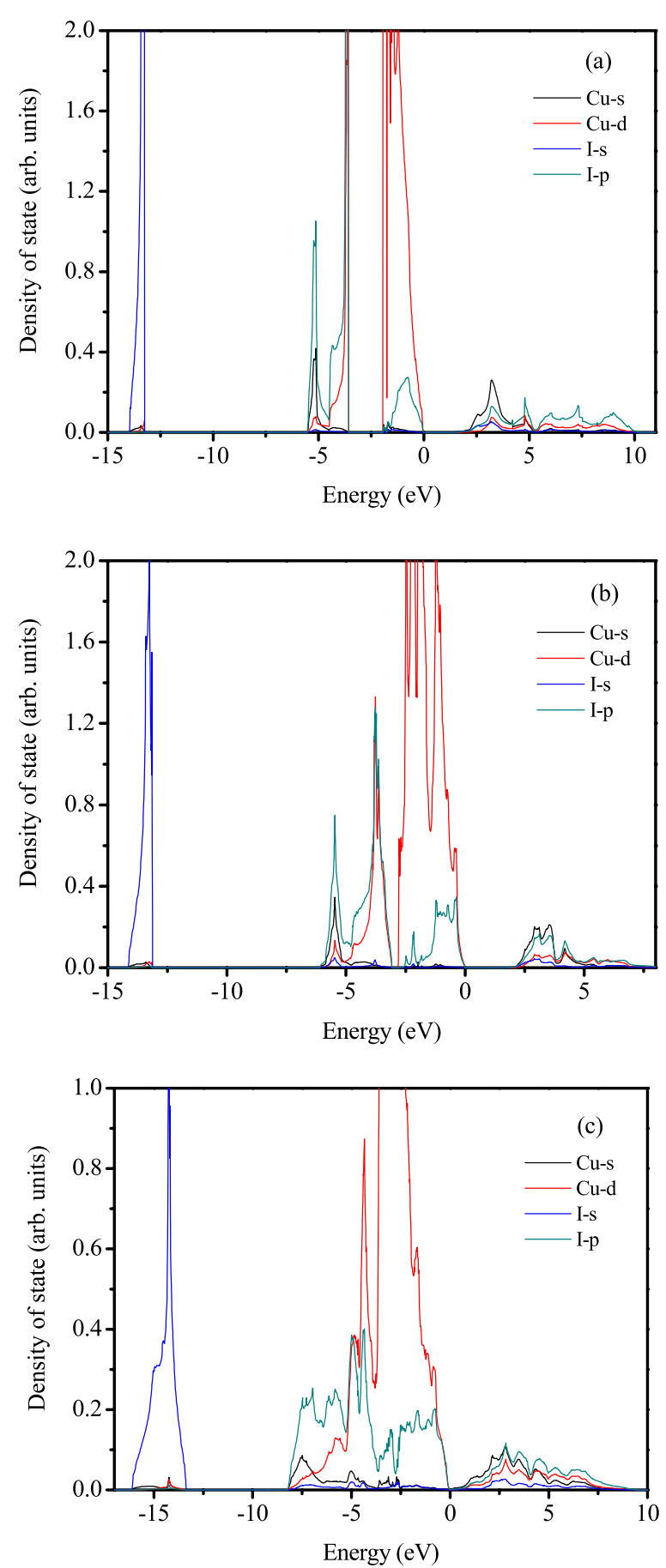

Figure 5. The projected density of states of CuI: (a) zinc-blende, (b) tetragonal, and (c) orthorhombic. Zero is aligned to the top of the valence band.

materials. Nevertheless distinct features in the band structures of the polymorphs are clearly demonstrated by the method employed here.

The calculated effective mass of electron and light and heavy hole at $\Gamma$ for polymorphs of $\mathrm{CuI}$ are also listed in table 3. We have fitted the energy dispersion of VBM (CBM) to a parabolic function in the vicinity of $\Gamma$ to calculate the hole (electron) effective mass. For the zinc-blende phase, the average effective masses of electron and heavy hole are
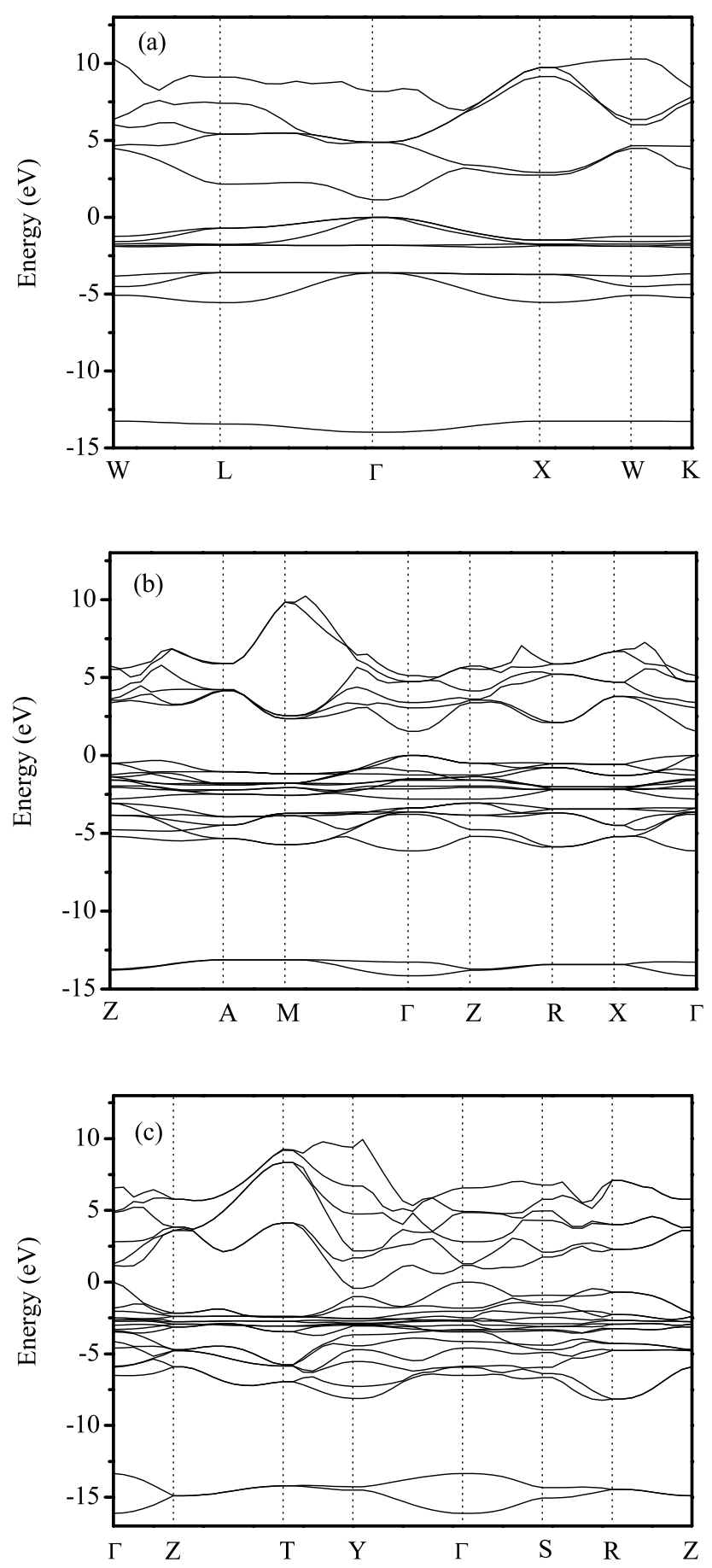

Figure 6. The calculated band structures of CuI: (a) zinc-blende, (b) tetragonal, and (c) orthorhombic. Zero is set as the top of the valence band.

in excellent agreement with the corresponding experimental values [28]. For example, the calculated value of the electron effective mass is 0.33 as compared to the corresponding experimental value of 0.34 . Note that the calculated values of the electron or hole effective mass mimic the anisotropy of the Brillouin zone, and are comparable to those associated with most of the ionic materials, suggesting that the electronic conduction in $\mathrm{CuI}$ is possible only if the electrons can be 
Table 2. Elastic properties of $\mathrm{CuI}$ in the zinc-blende, tetragonal and orthorhombic phases. Voigt, Reuss and Hill notation are labeled as V, R and $\mathrm{H}$.

\begin{tabular}{|c|c|c|c|c|c|c|}
\hline & \multicolumn{2}{|c|}{ Zinc-blende } & \multicolumn{2}{|c|}{ Tetragonal } & \multicolumn{2}{|c|}{ Orthorhombic } \\
\hline & This work & Expt $[24,25]$ & This work & Expt [8] & This work & Expt [22] \\
\hline \multicolumn{7}{|l|}{ Elastic constant (GPa) } \\
\hline$C_{11}$ & 50.4 & 45.1 & 78.8 & & 137.6 & \\
\hline$C_{12}$ & 33.5 & 30.7 & 24.7 & & 34.2 & \\
\hline$C_{13}$ & & & 40.3 & & 74.0 & \\
\hline$C_{22}$ & & & & & 185.8 & \\
\hline$C_{23}$ & & & & & 90.4 & \\
\hline$C_{33}$ & & & 56.2 & & 129.3 & \\
\hline$C_{44}$ & 20.4 & 18.5 & 0.6 & & 19.6 & \\
\hline$C_{55}$ & & & & & 73.3 & \\
\hline$C_{66}$ & & & 6.4 & & 58.4 & \\
\hline \multicolumn{7}{|l|}{ Bulk modulus (GPa) } \\
\hline$K_{V}$ & 39.1 & 35.5 & 47.2 & 50.0 & 94.4 & 115 \\
\hline$K_{R}$ & 39.1 & & 47.0 & & 92.1 & \\
\hline$K_{H}$ & 39.1 & & 47.1 & & 93.3 & \\
\hline \multicolumn{7}{|l|}{ Shear modulus (GPa) } \\
\hline$G_{V}$ & 15.6 & & 9.9 & & 47.2 & \\
\hline$G_{R}$ & 13.0 & & 2.3 & & 34.3 & \\
\hline$G_{H}$ & 14.3 & & 6.1 & & 40.7 & \\
\hline \multicolumn{7}{|l|}{ Elastic modulus (GPa) } \\
\hline$B_{X}$ & 117.4 & & 161.5 & & 197.2 & \\
\hline$B_{Y}$ & & & & & 314.6 & \\
\hline$B_{Z}$ & & & 112.4 & & 383.0 & \\
\hline Debye temperature $(\mathrm{K})$ & 175.0 & 165.0 & 115.8 & & 294.2 & \\
\hline
\end{tabular}

Table 3. Electronic properties of the zinc-blende, tetragonal and orthorhombic phases of CuI.

\begin{tabular}{llll}
\hline & Zinc-blende & Tetragonal & Orthorhombic \\
\hline Direct gap (eV) & 1.13 & 1.54 & Metallic \\
I 5s band width $(\mathrm{eV})$ & 0.72 & 1.02 & 2.78 \\
I 5p band width $(\mathrm{eV})$ & 5.54 & 6.13 & 8.24 \\
Separation I 5s-I 5p (eV) & 7.72 & 7.0 & 5.12 \\
Effective mass $\left(m_{\mathrm{e}}\right)$ & & & \\
Electron & $\Gamma-\mathrm{L}: 0.34$ & $\Gamma-\mathrm{Z}: 0.41$ & \\
& $\Gamma-\mathrm{X}: 0.33$ & $\Gamma-\mathrm{M}: 0.36$ & \\
Light hole & $\Gamma-\mathrm{L}: 0.38$ & $\Gamma-\mathrm{M}: 0.55$ & \\
& $\Gamma-\mathrm{X}: 0.50$ & & \\
Heavy hole & $\Gamma-\mathrm{L}: 1.14$ & $\Gamma-\mathrm{Z}: 1.45$ & \\
& $\Gamma-\mathrm{X}: 1.54$ & $\Gamma-\mathrm{M}: 1.22$ & \\
\hline
\end{tabular}

promoted to the conduction band by overcoming the relatively large band gap.

\subsection{Optical properties}

Electronic structure calculations can also predict the nature and location of the interband transitions in a crystal which will assist the experimentalists in identifying the peaks in the optical spectra obtained by either reflectance or photoelectron spectroscopy. The transition intensities are determined by the overlap of the wave function of initial and final state or transition matrix elements according to equation (2). According to the selection rules, the transitions of $\mathrm{p} \rightarrow \mathrm{s}$, $\mathrm{p} \rightarrow \mathrm{d}$ and $\mathrm{d} \rightarrow \mathrm{p}$ are allowed. The transition associated with $\mathrm{p} \rightarrow \mathrm{p}, \mathrm{d} \rightarrow \mathrm{s}$ and $\mathrm{d} \rightarrow \mathrm{d}$ are forbidden.

Figure 7 depicts the average dielectric function of $\mathrm{CuI}$ in the zinc-blende, tetragonal and orthorhombic phases. In the zinc-blende phase, the peaks in the imaginary part of dielectric function at 4.43, 6.00 and $6.86 \mathrm{eV}$ are associated with the interband transitions of $X_{3 v}-X_{1 c}, W_{2 v}-W_{1 c}$ and $K_{6 v}-\mathrm{K}_{1 c}$, respectively. The imaginary part of dielectric function of zinc-blende at $2.4 \mathrm{GPa}$ shows a blue shift compared to that at the ambient pressure (not presented). It is consistent to the results reported for $\mathrm{ZnO}$ [29]. In the tetragonal phase, the peaks at 3.90, 4.30, 5.35, 5.87 and $6.60 \mathrm{eV}$ are contributed from the transitions of $\Gamma_{3 v}-\Gamma_{2 c}, M_{7 v}-M_{1 c}, A_{1 v}-A_{3 c}, X_{5 v}-X_{1 c}$ and $Z_{11 v}-Z_{2 c}$, respectively. Compared to zinc-blende, the low energy absorption band at $1.86 \mathrm{eV}$ disappears due to the augment of band gap in the tetragonal CuI. For the orthorhombic phase, the interband transitions of $\Gamma_{8 v}-\Gamma_{2 c}$, $S_{7 v}-S_{1 c}$ and $Z_{9 v}-Z_{3 c}$ result in the peaks in the imaginary part of dielectric function at 4.26, 4.80 and $6.95 \mathrm{eV}$, respectively.

Table 4 lists the calculated dielectric constants and refractive indices of the $\mathrm{CuI}$ polymorphs. For the zinc-blende phase, calculated dielectric constant is 6.52 which is in excellent agreement with experimental value of 6.5 [24]. Note 
Table 4. Dielectric constants and refractive indices of polymorphs of $\mathrm{CuI}$.

\begin{tabular}{llll}
\hline & Zinc-blende & Tetragonal & Orthorhombic \\
\hline Dielectric constant & & & \\
$\quad$ This work & 6.52 & 6.63 & 12.32 \\
$\quad$ Experiment [24] & 6.5 & & \\
$\quad$ Previous study-theory [12] & 4.2 & & 3.51 \\
Refractive index & & 2.57 & \\
$\quad$ This work & 2.55 & & \\
$\quad$ Previous study-theory [12] & 2.05 & & \\
\hline
\end{tabular}
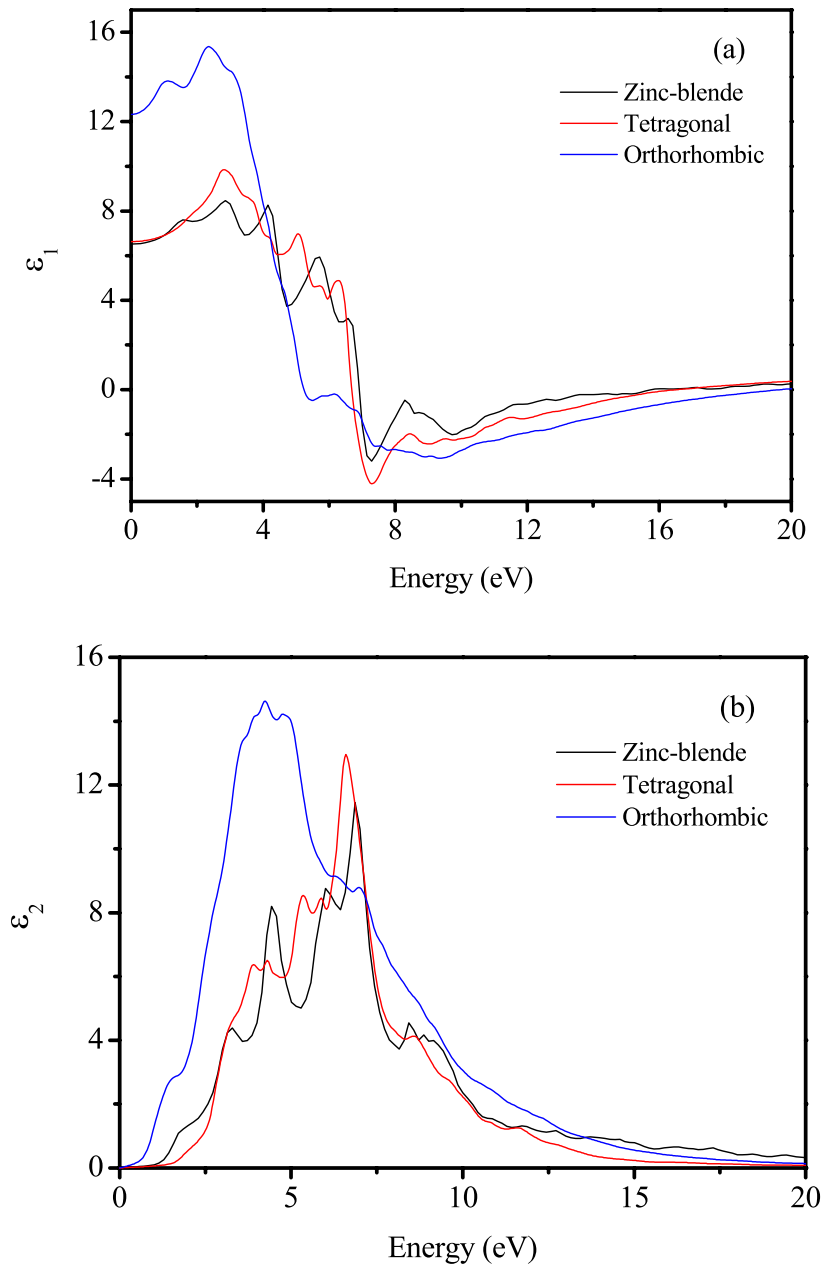

Figure 7. The calculated average (a) real part and (b) imaginary part of dielectric function of polymorphs of $\mathrm{CuI}$.

that the linear response of the system to an electromagnetic radiation is described by the dielectric function, which is directly related to the polarizability of the crystal. The polarizability, which represents the deformability of the electronic distribution, is to be connected with the shape of the valence charge density. Thus, a similarity in dielectric as well as optical properties between the zinc-blende and tetragonal phases is expected, as was predicted in the present study. A relatively large dielectric constant of $\mathrm{CuI}$ in the high-pressure orthogonal phase is likely to be due to the denser packing of ions and higher coordination number in the lattice.

\section{Summary}

The results of a comprehensive investigation of the polymorphs of $\mathrm{CuI}$ are reported. The calculated structural, elastic and optical properties of the zinc-blende $\mathrm{CuI}$ are in excellent agreement with the corresponding experimental values. The zinc-blende and tetragonal $\mathrm{CuI}$ are direct gap semiconductors and their optical properties are similar to each other. The orthorhombic $\mathrm{CuI}$ becomes metallic under high pressure. The valence band is almost flat indicating a rather large effective mass for holes for the polymorphs of $\mathrm{CuI}$ considered.

It has been suggested that $\mathrm{CuI}$ makes a transition to the rhombohedral phase for pressures $<1.4 \mathrm{GPa}$ [8-10]. This is in contrast to what has been predicted by our calculations based on density functional theory. We find a negative value of $B_{Z}$ for the rhombohedral phase suggesting its mechanical instability. We also note that the rhombohedral phase does not exist for the other I-VII compounds such as AgI and $\mathrm{CuBr}$ [30]. A new experimental study is therefore warranted to confirm our predictions for the high-pressure polymorphs of $\mathrm{CuI}$.

\section{Acknowledgments}

Jiajie Zhu acknowledges the hospitality of Michigan Technological University. This work is funded by the National Natural Science Foundation of China (Grant nos 91022002, 11179019) and the Significant National Special Project of the Ministry of Science and Technology of China for Development of Scientific Instrument and Equipment (Grant no. 2011YQ13001902).

\section{References}

[1] Adipranoto D S, Shikanai F, Yonemura M, Mori K, Park J G, Itoh K and Kamiyama T 2009 Solid State Ion. 180 492-6

[2] Lewonczuk S, Ringeissen J, Beaurepaire E and Khan M A 1994 Phys. Rev. B 49 2344-50

[3] Ichida H, Kanematsu Y, Shimomura T, Mizoguchi K, Kim D and Nakayama M 2005 Phys. Rev. B 72045210

[4] Perera V P S and Tennakone K 2003 Sol. Energy Mater. Sol. Cells 79 249-55

[5] Derenzoa S E, Weber M J and Klintenberg M K 2002 Nucl. Instrum Methods Phys. Res. A $486214-9$

[6] Gao P, Gu M, Liu X, Liu B and Huang S 2009 Appl. Phys. Lett. 95221904 
[7] Sekkal W, Zaoui A, Laref A, Certier M and Aourag H 2000 J. Phys.: Condens. Matter 12 6173-82

[8] Hull S and Keen D A 1994 Phys. Rev. B 50 5868-85

[9] Blacha A, Christensen N E and Cardona M 1986 Phys. Rev. B 33 2413-21

[10] Ves S, Glotzel D, Cardona M and Overhof H 1981 Phys. Rev. B 24 3073-85

[11] Hernandez-Cocoletzi H, Cocoletzi G H, Rivas-Silva J F, Flores A and Takeuchi N 2009 J. Nano Res. 5 25-30

[12] Amrani B, Benmessabih T, Tahiri M, Chiboub I, Hiadsi S and Hamdache F 2006 Physica B 381 179-86

[13] Singh R K and Gupta D C 1989 Phys. Rev. B 40 11278-83

[14] Yuce G, Çolakolu K, Deligoz E and Çiftci Y O 2007 AIP Conf. Proc. 899674

[15] Groh D, Pandey R, Sahariah M B, Amzallag E, Baraille I and Rerat M 2009 J. Phys. Chem. Solids 70 789-95

[16] Kresse G and Furthmuller J 1996 Phys. Rev. B 54 11169-86

[17] Kresse G and Joubert D 1999 Phys. Rev. B 59 1758-75

[18] Perdew J P, Burke K and Ernzerhof M 1996 Phys. Rev. Lett. 77 3865-8
[19] Groh D, Slough W J, Pandey R, Karna S P and Dandekar D 2011 Phys. Rev. B 83115122

[20] Gajdos M, Hummer K, Kresse G, Furthmüller J and Bechstedt F 2006 Phys. Rev. B 73045112

[21] Sampath S K, Kanhere D G and Pandey R 1999 J. Phys.: Condens. Matter 11 3635-44

[22] Hofmann M, Hull S and Keen D A 1995 Phys. Rev. B 51 12022-5

[23] Baghmar D, Gaur N K and Gupta D C 2010 Physica B 405 133-9

[24] Hanson R C, Hallberg J R and Schwab C 1972 Appl. Phys. Lett. 21 490-2

[25] Vardeny Z, Gilat G and Moses D 1978 Phys. Rev. B 18 4487-96

[26] Ravindran P, Fast L, Korzhavyi P A, Johansson B and Eriksson O 1988 J. Appl. Phys. 84 4891-904

[27] Wu Z, Zhao E, Xiang H, Hao X, Liu X and Meng J 2007 Phys. Rev. B 76054115

[28] Wang J, Li J and Li S 2011 J. Appl. Phys. 110054907

[29] Sun J, Wang H, He J and Tian Y 2005 Phys. Rev. B 71125132

[30] Keen D A and Hull S 1993 J. Phys.: Condens. Matter 5 23-32 